\title{
Is health-related quality of life associated with adequacy of hemodialysis in chronic kidney disease patients?
}

Lamia M. Hasan', Dina A. H. Shaheen², Ghada A. H. El Kannishy', Nagy A. H. Sayed-Ahmed' and Ahmed M. Abd El Wahab ${ }^{1 *}$

\begin{abstract}
Introduction: Monitoring Health Related Quality of Life (HRQoL) in different stages of chronic kidney disease is advised by all nephrology societies. We aimed to study the relation between quality of life and dialysis adequacy.

Methods: One hundred patients (51\% males), on regular hemodialysis 3/week for $>6$ months in two hospitals were included. Single pool Kt $/ \mathrm{N}$ was used to assess dialysis adequacy. Patients were grouped into 3 divisions according to $\mathrm{Kt} / \mathrm{v}$ : Group A $>1.5(n=24)$, group B 1.2-1.5 $(n=54)$ and group C $<1.2(n=22)$. KDQOL-SF ${ }^{\mathrm{TM}}$ questionnaire was used to study quality of life in our groups. Group $C$ was reassessed after 3 months of improving Kt/v.

Results: Mean values were: Kt/N (1.48 \pm 0.41$)$, Cognitive Function (84.27 \pm 9.96$)$, Work status (30.00 \pm 33.33$)$, Energy (45.70 \pm 13.89 ), Physical Function and Role limitations due to physical function ( $45.30 \pm 12.39$ and $31.25 \pm 19.26$, respectively). Group A had significantly higher scores of KDQOL-SF except Role limitations due to Physical Function. All subscales improved in group C after Kt/v improvement except 3 subscales, namely, work status, patient satisfaction and role limitation due to physical and emotional functions.
\end{abstract}

Conclusion: Inadequate HD badly affects quality of life and improving adequacy refines many components of quality of life.

Keywords: Hemodialysis, HRQoL, Dialysis adequacy, KDQOL-SF

\section{Introduction}

End stage renal disease (ESRD) is an increasing issue worldwide impairing patients Qol [1]. Hemodialysis (HD) is the mostly used for treatment [2] leading to overt changes in patients' lives $[3,4]$.

Improving dialysis adequacy, anemia, hyperparathyroidism and many other factors is known to lessen morbidity and mortality in dialysis patients [5-8].

*Correspondence: drabdoo34@gmail.com

1 Mansoura Nephrology and Dialysis Unit (MNDU), Mansoura University Hospital, Mansoura, Egypt

Full list of author information is available at the end of the article
Dialysis adequacy evaluation is difficult as many factors like volume status, electrolytes and acid base interfere with it but Kt/v is the mostly used parameter.

The reported prevalence of ESRD in Egypt is estimated to be 624 pmp [9].

Many physicians looking after hemodialysis patients are interested mainly in the clinical and laboratory data of their patients, paying little attention to the patients' emotional satisfaction and their quality of life.

Most of regular HD patients are vulnerable for many physical and psychological disabilities like easy fatigability, myalgia, depression and sexual dysfunction [10].

Many disease-specific questionnaires such as the World Health Organization Quality of Life Survey 
(WHOQoL), Short Form (SF)-36 health questionnaire and the Choices Health Experiences Questionnaire [CHEQ] are used to assess HRQoL in ESRD [11].

Of the 36 items in the SF-36 questionnaire, we only used 35 items representing 2 summary measures, the physical component summary (PCS), mental component summary (MCS) and 8 scales [12].

Advantages of KDQOL-SF are: 1) Tested in different populations with renal disease. 2) It includes general and specific modules evaluating chronic kidney disease. 3) Effective in hemodialysis and peritoneal dialysis patients' assessment. 4) It can be self-applied or applied by an interviewer. 5) It has been culturally adapted and validated in various languages [13].

This work was planned to focus on assessing HRQOL among ESRD patients treated with hemodialysis in relation to different variables, especially the adequacy of dialysis relying on the calculated $\mathrm{kt} / \mathrm{V}$.

\section{Patients and methods}

\section{Study}

This study was conducted in Mansoura Nephrology and Dialysis Unit (MNDU) and Talkha hemodialysis units from 2017 to 2018.

Criteria of inclusion:

- Gender: Both

- Age: Older than 20 years

- Maintained on HD (thrice weekly) for $\geq 6$ months.

- Patients with controlled DM or hypertension (HTN) (Uncontrolled blood pressure $>150 / 90$ in average weekly predialysis reading while the patient is on maximum doses of 3 or more antihypertensive medications with ideal dry body weight. Uncontrolled DM means FPG > 130 and PPG > 150 in average weekly reading using bedside glucose measuring devices).

The exclusion criteria included:

- Recently dialyzed patients (less than 6 months)

- Patients with cognitive impairment

- Patients with mental retardation

- Patients with COPD

- Patients with severe anaemia

- Patient with uncontrolled DM or HTN

- Patients with other system failure

The study was approved by our faculty IRB, The nature and intent of the study were fully explained to all subjects, and an informed written consent was obtained from each participant before running the study.
One hundred thirty-four patients were screened and 119 patients met the inclusion criteria. One hundred patients out of 119 agreed to participate.

All patients were being subjected to history taking and full examination.

\section{Study design}

The 100 subjects who agreed to participate in this study were divided according to their $\mathrm{kt} / \mathrm{v}$ into three groups:

The first group (group A) with $\mathrm{kt} / \mathrm{v}>1.5(n=24)$.

The second group (group B) with kt/v range from [1.5-1.2] ( $n=54)$.

The third group (group c) with $\mathrm{kt} / \mathrm{v}<1.2(n=22)$.

Measures to improve kt/v were applied to Group C and then all the collected parameters were tested again in this group.

Improving $\mathrm{kt} / \mathrm{v}$ is done for group $\mathrm{C}$ for 3 months through:

1. Adjustment of dialyser surface area

2. Increasing blood flow rates.

3. Extending session time.

\section{Anthropometric measurements}

The patients' weight in kilograms before and after dialysis sessions, dialysis session duration, ultrafiltration volume, was collected for all patients.

\section{Specimen collection}

Pre-dialysis blood samples were withdrawn into 2 vaccutainer tubes from all subjects at the start of the study and after 3 months for group $\mathrm{C}$. Both predialysis and postdialysis urea samples were withdrawn before and after the same session of HD. Daugirdas et al. [14] formula was used for Calculation of $\mathrm{Kt} / \mathrm{V}$ :

$\mathrm{Kt} / \mathrm{V}=-\ln ($ Ratio-0.03) $+[(4-(3.5 \times$ Ratio $)) \times(\mathrm{UF} / \mathrm{Wt})]$, in which Ratio is post BUN/pre BUN, UF is ultrafiltration volume, and Wt is post dialysis weight.

Biochemical analysis:

- Complete blood count, Liver function tests, serum calcium, serum phosphorus and $\mathrm{iPTH}$ were measured.

\section{Subjective methods}

HRQOL was assessed with the validated Kidney Disease Quality of Life-Short Form (KDQOL-SF version 1.3): http://gim.med.ucla.edu/kdqol/downloads/-download. html [15]. 
KDQOL-SF 36 issued by Hays et al. in 1994 and is published for free on internet website: http://gim.med.ucla. edu/kdqol/downloads-download.html.

For purpose of simplicity, the KDQOL-SF version 1.3 was summarized in three categories; namely, kidney disease targeted scales, physical component and mental component summaries, each of which drives its core information and calculations from one or a group of questions from the original 36 questions. The user manual of this questionnaire with these details was previously published [16].

The KDQOL-SF can be split in a generic part and a disease-specific part. First, the generic part is formed by the SF-36 version 1 . The domains of the SF-36 can be summarized in two summary scores, one for physical functioning (physical component summary-PCS) and one for mental functioning (mental component summaryMCS). These summaries are constructed so that a score of 50 represents the mean of the general United States population with a standard deviation of 10 [16]. Second, the disease-specific part of the KDQOL-SF consists of 44 kidney disease-targeted questions. The responses to these items are condensed in 12 domains. These domains have a score from 0 to 100 , with higher scores indicating the absence of problems.

Results from the SF-36 instrument are further summarized into a physical composite summary (PCS) score and a mental composite summary (MCS) score. PCS aggregates items from physical function, role physical, pain, vitality and general health. MCS aggregates items from role emotional, emotional wellbeing, energy, social function and general health. These summaries are constructed so that a score of 50 represents the mean of the general United States population with a standard deviation of 10 [16].

There are some explanations to some questionnaire items:

1. Physical functioning (PF) - the level of limitation of physical activity caused by health limitations

2. Role physical (RP) - helps measure the limitations of patient-specific physical activity caused by health problems

3. Vitality (VT) - measurement of energy and fatigue

4. Social functioning (SF) - defines the level of social life limitations caused by physical and emotional discomfort

5. Mental health $(\mathrm{MH})-$ defines the level of psychological stress and well-being [17].

A previous study, carried out in Alexandria, Egypt [18], utilized an Arabic translation of the KDQOL-SF version 1.3 and the authors concluded that this translation is a validated and reliable tool for studying the HRQOL in ESRD patients. In the current study, this Arabic version of KDQOL-SF1.3 was also used for assessment of HRQOL with some additional section translated from the original English version to suit the patients on regular hemodialysis. The questionnaire was used to assess the studied population and to group $\mathrm{C}$ before and after improving $\mathrm{kt} / \mathrm{v}$. One item studying sexual activity in the KDQOL-SF ${ }^{\mathrm{TM}}$ was excluded from the study as nearly all patients refused to talk about that issue (cultural concerns).

\section{Scoring system}

The standard scoring program of the KDQOL-SF ${ }^{\mathrm{TM}} 1.3$ is based on the Microsoft Excel 97 spreadsheet program and includes information about the computation method:

https://www.rand.org/health-care/surveys_tools/ kdqol.html.

The scores for each dimension range from 0 to 100 , with higher scores reflecting better HRQOL. The change in health (question 2) of the SF36 scale and the 0-10 overall health rating (question 22) items are scored as single items [13].

\section{Statistical methods}

Data were tabulated, coded then analyzed using the computerprogram SPSS (Statistical package for social science) version 20.0 to obtain.

Descriptive data:

Descriptive statistics were calculated in the form of:

1. Mean \pm Standard deviation (SD).

2. Median \& interquartile range (IQR) $[25,75]$.

3. Frequency (Number-percent).

\section{Analytical statistics}

- In the statistical comparison between the different groups the normality of distribution in continuous data was checked by kolmogorov-smirnov test, and Shapiro-Wilk normality test

- The significance of difference in groups (A, B, C) was tested using Kruscalwalis' H test

- The comparison between the three groups was done using Bonferroni post-hoc test. The level of significance was set at $p<0.05$.

- Measurements within the same group $C$ before and after improving, were statistically compared using paired t-test or Wilcoxon signed-ranks test for parametric or non-parametric data, respectively. Results are presented as counts for nominal variables, and as mean \pm standard deviation (SD) and median (inter- 
quartile range) for continuous variables. The level of significance was set at $p<0.05$.

\section{Results}

This study involved 100 HD patients with an age ranging between 28 and 62 years (mean $48.8 \pm 5.89$ years). DM was present in $26 \%$ of the patients while HTN was present in $82 \%$. HTN was the cause of ESRD in $58 \%$ of the patients while DM was the cause in $22 \%$ (Table 1). It included 51 males and 49 females with their weights ranging between 54 and $104 \mathrm{Kg}$ (mean $79.01 \pm 11.35$ $\mathrm{Kg}$ ). All the patients were treated with regular HD sessions three times weekly with a median (IQR) duration of $4 \mathrm{~h}$ and the interdialytic weight gain ranging between

Table 1 General descriptive characteristics of the total studied patients $(n=100)$

\begin{tabular}{ll}
\hline Age (Year, Mean \pm SD) & $\mathbf{4 8 . 8} \pm \mathbf{5 . 8 9}$ \\
\hline DM (N, \%) & $26(26 \%)$ \\
HTN (N, \%) & $82(82 \%)$ \\
Original kidney disease & \\
• HTN (N, \%) & $58(58 \%)$ \\
• DM (N, \%) & $22(22 \%)$ \\
• Stones, pyelonephritis & $12(12 \%)$ \\
• Undetermined & $8(8 \%)$ \\
\hline
\end{tabular}

1 and $4 \mathrm{~kg}$ (mean $2.60 \pm 0.96)$ per session. The mean $\mathrm{kt} / \mathrm{v}$ was $1.48 \pm 41$. As regard the KDQOL_SF v 1.3 scoring system, the mean of the scores of the item related to "Symptoms and Problem" list of the total studied population was $71.29 \pm 17.27$; a figure that is not far from a maximum possible score of 100 for the least symptoms and problems. The mean of the scores of the item dealing with "Effect of kidney disease" was also reasonable, $65.13 \pm 11.03$. On the other hand, the mean of the scores of the item dealing with "Work status", "Energy", "Physical Function" as well as "Role limitations due to physical function" were very low $(30.00 \pm 33.33,45.70 \pm 13.89$, $45.30 \pm 12.39$ and $31.25 \pm 19.26$; respectively), while the mean of the scores of the item referring to "Cognitive Function" was high [mean 84.27 with SD 9.96] (Table 2). Twenty four patients of the studied population had $\mathrm{kt} / \mathrm{v}>1.5$ (group A), 54 patients had kt/v between (1.2 and 1.5; group B) and 22 had $\mathrm{Kt} / \mathrm{v}<1.2$ (group C). The studied general and laboratory variables of the three $\mathrm{Kt} / \mathrm{v}$ groups (Table 3) were comparable regarding age, pre-dialysis urea and albumin. However, the body weights before sessions were significantly higher in group (A). There was also a highly significant difference between the interdialytic weight gain and session durations between the three groups with the greatest volumes and longest sessions in group (A). On the other hand, calcium and hemoglobin were significantly higher in group A, while PTH and phosphorus were significantly higher in group $C$ versus other groups (Table 3). Regarding the KDQOL-SF V 1.3

Table 2 Descriptives of the response to the questionnaire of the total studied patients $(n=100)$

\begin{tabular}{lll}
\hline Questionnaire's items & Mean \pm SD & Median (IQR) \\
\hline Symptoms / problems list & $71.29 \pm 17.27$ & $77.08(70.83-81.25)$ \\
Effect of kidney disease & $65.13 \pm 11.03$ & $65.63(95.37-71.87)$ \\
Burden of kidney disease & $55.75 \pm 15.78$ & $62.50(50-68.75)$ \\
Work Status & $30.00 \pm 33.33$ & $25(0.00-50.00)$ \\
Cognitive function & $84.27 \pm 9.96$ & $86.67(80.00-93.33))$ \\
Quality of Social interaction & $64.27 \pm 14.63$ & $66.67(53.33-73.33)$ \\
Sleep & $64.25 \pm 18.34$ & $70(58.13-75)$ \\
Overall health & $65.50 \pm 20.76$ & $70.00(70-80)$ \\
Patient satisfaction & $44.33 \pm 11.42$ & $50.00(33.33-50)$ \\
Physical function & $45.30 \pm 12.39$ & $45.00(35.0-53.75)$ \\
Role limitation due to physical function & $31.25 \pm 19.26$ & $25.00(25.0-50)$ \\
Pain & $49.90 \pm 20.67$ & $55.00(36.87-67.50)$ \\
General health & $52.40 \pm 11.84$ & $50.00(45-60)$ \\
Emotional Well Being & $61.36 \pm 14.17$ & $68.00(56.00-72)$ \\
Role limitations due to emotional problems & $52.67 \pm 28.10$ & $66.67(33.33-66.66)$ \\
Social function & $54.20 \pm 21.84$ & $62.5(50.00-75.00)$ \\
Energy & $45.70 \pm 13.89$ & $50.00(40.00-55.00))$ \\
Physical composite & $36.60 \pm 5.51$ & $36.70(32.47-40.20)$ \\
Mental composite & $42.11 \pm 9.11$ & $45.20(36.11-48.23)$ \\
\hline
\end{tabular}


Table 3 Comparison between the three groups of patient regarding the general data $(n=100)$

\begin{tabular}{|c|c|c|c|c|c|c|c|c|}
\hline & & Group A & Group B & Group C & $P$ value. & $\begin{array}{l}\text { Group A vs } \\
\text { Group B }\end{array}$ & $\begin{array}{l}\text { Group A vs } \\
\text { Group C }\end{array}$ & $\begin{array}{l}\text { Group B vs } \\
\text { Group C }\end{array}$ \\
\hline \multirow[t]{3}{*}{ Age (Year) } & $N$ & 24 & 54 & 22 & 0.636 & & & \\
\hline & Mean & $50.45 \pm 1.77$ & $49.89 \pm 2.38$ & $50.64 \pm 3.84$ & & & & \\
\hline & Mean Ranks & 53.79 & 47.98 & 53.09 & & & & \\
\hline \multirow[t]{3}{*}{ Pre-urea (mg/dl) } & $N$ & 24 & 54 & 22 & 0.206 & & & \\
\hline & Mean & $120.33 \pm 28.80$ & $129.93 \pm 26.35$ & $129.00 \pm 35.17$ & & & & \\
\hline & Mean Ranks & 41.44 & 53.94 & 51.95 & & & & \\
\hline \multirow{3}{*}{$\begin{array}{l}\text { Pre session } \\
\text { weight }(\mathrm{Kg})\end{array}$} & N & 24 & 54 & 22 & 0.042 & 0.036 & 0.389 & 1.000 \\
\hline & Mean & $83.48 \pm 10.54$ & $76.99 \pm 11.10$ & $79.09 \pm 13.99$ & & & & \\
\hline & Mean Ranks & 62.98 & 45.11 & 50.11 & & & & \\
\hline \multirow{3}{*}{$\begin{array}{l}\text { Interdialytic } \\
\text { weight gain (Kg) }\end{array}$} & $\mathrm{N}$ & 24 & 54 & 22 & 0.002 & 1.000 & 0.006 & 0.003 \\
\hline & Mean & $2.35 \pm 1.03$ & $2.42 \pm 0.95$ & $3.20 \pm 0.59$ & & & & \\
\hline & Mean Ranks & 43.44 & 45.90 & 69.50 & & & & \\
\hline \multirow{3}{*}{$\begin{array}{l}\text { Dialysis session } \\
\text { time (hrs) }\end{array}$} & $N$ & 24 & 54 & 22 & $<0.0001$ & 0.938 & $<0.0001$ & $<0.0001$ \\
\hline & Mean & $4.00 \pm 0.00$ & $3.89 \pm 0.32$ & $3.05 \pm 0.043$ & & & & \\
\hline & Mean Ranks & 63.50 & 58.00 & 17.91 & & & & \\
\hline \multirow{3}{*}{$\begin{array}{l}\text { Serum calcium } \\
(\mathrm{mg} / \mathrm{dl})\end{array}$} & $N$ & 24 & 54 & 22 & 0.001 & 0.010 & 0.001 & 0.568 \\
\hline & Mean & $9.84 \pm 0.83$ & $9.22 \pm 0.79$ & $8.93 \pm 0.88$ & & & & \\
\hline & Mean Ranks & 68.50 & 47.59 & 38.00 & & & & \\
\hline \multirow{3}{*}{$\begin{array}{l}\text { Serum Ph (mg/ } \\
\text { dl) }\end{array}$} & N & 24 & 54 & 21 & 0.007 & 1.000 & 0.013 & 0.015 \\
\hline & Mean & $4.65 \pm 0.57$ & $4.68 \pm 1.08$ & $5.38 \pm 0.77$ & & & & \\
\hline & Mean Ranks & 42.73 & 46.56 & 67.17 & & & & \\
\hline \multirow{3}{*}{$\begin{array}{l}\text { Serum albumin } \\
(g / d l)\end{array}$} & N & 24 & 54 & 22 & 0.704 & & & \\
\hline & Mean & $3.35 \pm 0.40$ & $3.27 \pm 0.39$ & $3.28 \pm 0.46$ & & & & \\
\hline & Mean Ranks & 54.79 & 49.00 & 49.50 & & & & \\
\hline \multirow{3}{*}{$\begin{array}{l}\text { Serum PTH (pg/ } \\
\text { ml) }\end{array}$} & $N$ & 24 & 53 & 22 & $<0.0001$ & 0.480 & $<0.0001$ & $<0.0001$ \\
\hline & Mean & $158.10 \pm 150.73$ & $318.89 \pm 486.23$ & $750.00 \pm 523.20$ & & & & \\
\hline & Mean Ranks & 35.25 & 45.18 & 77.70 & & & & \\
\hline \multirow[t]{3}{*}{$\mathrm{Hb}(\mathrm{g} / \mathrm{dl})$} & $N$ & 24 & 54 & 22 & $<0.0001$ & 0.012 & $<0.0001$ & 0.059 \\
\hline & Mean & $10.20 \pm 1.05$ & $9.71 \pm 0.86$ & $9.10 \pm 0.78$ & & & & \\
\hline & Mean Ranks & 69.81 & 49.34 & 32.27 & & & & \\
\hline \multirow[t]{2}{*}{$\mathrm{Kt} / \mathrm{V}$} & $N$ & 24 & 54 & 22 & $<0.0001$ & $<0.0001$ & $<0.0001$ & $<0.0001$ \\
\hline & Mean Ranks & 88.50 & 49.50 & 11.50 & & & & \\
\hline
\end{tabular}

$P$ value is calculated by Kruskal-Wallis $\mathrm{H}$ test

Comparison between three groups is calculated by post-hoc test

questionnaire, the comparison of the scores of the items between the three studied groups, showed highly significant differences between the three groups except for 2 subscales; the "Role limitations due to Physical and emotional function" (Table 4).

A significant difference was found regarding presession weight and interdialytic weight gain after improvement of kt/v in group C. (Table 5). There was a statistically significant difference regarding both dialysis session duration and PTH; with lower PTH values and longer dialysis sessions duration in patients with improved Kt/v (Table 6). Post dialysis urea, phosphorous and hemoglobin were significantly changed in group $\mathrm{C}$ after improvement of kt/v (Table 7).

All the studied subscales showed significant change after improvement of kt/v except for Work status, Patient satisfaction, Role limitation for physical and Role limitation due to emotional problems (Table 8).

Regarding linear study of $\mathrm{Kt} / \mathrm{v}$ and the summarizing domains of SF-36 (physical and mental composites), the correlation of $\mathrm{Kt} / \mathrm{v}$ and these domains controlled for two important correlated confounders, namely Hemoglobin and PTH showed statistically significant positive correlation. When Work status was correlated to $\mathrm{Kt} / \mathrm{v}$ 
Table 4 Analysis of the subscales of the questionnaire in the studied groups $(n=100)$

\begin{tabular}{|c|c|c|c|c|c|c|}
\hline Questionnaire's items & Kt/V Groups & $\mathbf{N}$ & Mean Rank & $P$ value* & & $P$ value ${ }^{* *}$ \\
\hline \multirow[t]{3}{*}{ Symptoms / problems list } & Group-A & 24 & 77.08 & $<0.0001$ & Group-A vs Group-B & 0.004 \\
\hline & Group-B & 54 & 54.57 & & Group-A vs Group-C & $<0.0001$ \\
\hline & Group-C & 22 & 11.5 & & Group-B vs Group-C & $<0.0001$ \\
\hline \multirow[t]{3}{*}{ Effect of kidney disease } & Group-A & 24 & 81.9 & $<0.0001$ & Group-A vs Group-B & $<0.0001$ \\
\hline & Group-B & 54 & 52.31 & & Group-A vs Group-C & $<0.0001$ \\
\hline & Group-C & 22 & 11.8 & & Group-B vs Group-C & $<0.0001$ \\
\hline \multirow[t]{3}{*}{ Burden of kidney disease } & Group-A & 24 & 71.94 & $<0.0001$ & Group-A vs Group-B & 0.095 \\
\hline & Group-B & 54 & 56.86 & & Group-A vs Group-C & $<0.0001$ \\
\hline & Group-C & 22 & 11.5 & & Group-B vs Group-C & $<0.0001$ \\
\hline \multirow[t]{3}{*}{ Work Status } & Group-A & 24 & 44.25 & 0.003 & Group-A vs Group-B & 1.000 \\
\hline & Group-B & 54 & 46.61 & & Group-A vs Group-C & 0.086 \\
\hline & Group-C & 22 & 66.86 & & Group-B vs Group-C & 1.000 \\
\hline \multirow[t]{3}{*}{ Cognitive Function } & Group-A & 24 & 70.19 & $<0.0001$ & Group-A vs Group-B & 0.122 \\
\hline & Group-B & 54 & 56.06 & & Group-A vs Group-C & $<0.0001$ \\
\hline & Group-C & 22 & 15.39 & & Group-B vs Group-C & $<0.0001$ \\
\hline \multirow[t]{3}{*}{ Quality of Social interaction } & Group-A & 24 & 40.27 & $<0.0001$ & Group-A vs Group-B & $<0.0001$ \\
\hline & Group-B & 54 & 69.23 & & Group-A vs Group-C & 0.011 \\
\hline & Group-C & 22 & 15.68 & & Group-B vs Group-C & $<0.0001$ \\
\hline \multirow[t]{3}{*}{ Sleep } & Group-A & 24 & 79.35 & $<0.0001$ & Group-A vs Group-B & 0.001 \\
\hline & Group-B & 54 & 53.56 & & Group-A vs Group-C & $<0.0001$ \\
\hline & Group-C & 22 & 11.5 & & Group-B vs Group-C & $<0.0001$ \\
\hline \multirow[t]{3}{*}{ Overall Health } & Group-A & 24 & 69.88 & $<0.0001$ & Group-A vs Group-B & 0.222 \\
\hline & Group-B & 54 & 57.78 & & Group-A vs Group-C & $<0.0001$ \\
\hline & Group-C & 22 & 11.5 & & Group-B vs Group-C & $<0.0001$ \\
\hline \multirow[t]{3}{*}{ 1.1.Patient Satisfaction } & Group-A & 24 & 58.88 & 0.001 & Group-A vs Group-B & 0.021 \\
\hline & Group-B & 54 & 41.43 & & Group-A vs Group-C & 1.000 \\
\hline & Group-C & 22 & 63.64 & & Group-B vs Group-C & 0.003 \\
\hline \multirow[t]{3}{*}{ Physical Function } & Group-A & 24 & 66.23 & $<0.0001$ & Group-A vs Group-B & 0.071 \\
\hline & Group-B & 54 & 57.45 & & Group-A vs Group-C & $<0.0001$ \\
\hline & Group-C & 22 & 16.27 & & Group-B vs Group-C & $<0.0001$ \\
\hline \multirow[t]{3}{*}{ Role limitation due to Physical function } & Group-A & 24 & 56.96 & 0.39 & Group-A vs Group-B & \\
\hline & Group-B & 54 & 50.55 & & Group-A vs Group-C & \\
\hline & Group-C & 22 & 43.34 & & Group-B vs Group-C & \\
\hline \multirow[t]{3}{*}{ General Health } & Group-A & 24 & 75.79 & $<0.0001$ & Group-A vs Group-B & $<0.0001$ \\
\hline & Group-B & 54 & 37.89 & & Group-A vs Group-C & 0.001 \\
\hline & Group-C & 22 & 53.86 & & Group-B vs Group-C & 0.018 \\
\hline \multirow[t]{3}{*}{ Pain } & Group-A & 24 & 74.75 & $<0.0001$ & Group-A vs Group-B & 0.011 \\
\hline & Group-B & 54 & 55.45 & & Group-A vs Group-C & $<0.0001$ \\
\hline & Group-C & 22 & 11.6 & & Group-B vs Group-C & $<0.0001$ \\
\hline \multirow[t]{3}{*}{ Emotional Well Being } & Group-A & 24 & 70.92 & 0.001 & Group-A vs Group-B & 0.171 \\
\hline & Group-B & 54 & 57.31 & & Group-A vs Group-C & $<0.0001$ \\
\hline & Group-C & 22 & 11.5 & & Group-B vs Group-C & $<0.0001$ \\
\hline \multirow[t]{3}{*}{ 1.1. Role limitations due to emotional function } & Group-A & 24 & 64.88 & 0.26 & Group-A vs Group-B & \\
\hline & Group-B & 54 & 53.96 & & Group-A vs Group-C & \\
\hline & Group-C & 22 & 26.32 & & Group-B vs Group-C & \\
\hline \multirow[t]{3}{*}{ Social Functions } & Group-A & 24 & 80.35 & $<0.0001$ & Group-A vs Group-B & $<0.0001$ \\
\hline & Group-B & 54 & 52.58 & & Group-A vs Group-C & $<0.0001$ \\
\hline & Group-C & 22 & 12.82 & & Group-B vs Group-C & $<0.0001$ \\
\hline
\end{tabular}


Table 4 (continued)

\begin{tabular}{|c|c|c|c|c|c|c|}
\hline Questionnaire's items & Kt/V Groups & $\mathrm{N}$ & Mean Rank & $P$ value* & & $P$ value*** \\
\hline \multirow[t]{3}{*}{ Energy } & Group-A & 24 & 74.5 & $<0.0001$ & Group-A vs Group-B & 0.042 \\
\hline & Group-B & 54 & 55.72 & & Group-A vs Group-C & $<0.0001$ \\
\hline & Group-C & 22 & 11.5 & & Group-B vs Group-C & $<0.0001$ \\
\hline \multirow[t]{3}{*}{ Physical Composite } & Group-A & 24 & 65.92 & 0.002 & Group-A vs Group-B & 0.042 \\
\hline & Group-B & 54 & 51.33 & & Group-A vs Group-C & 0.001 \\
\hline & Group-C & 22 & 31.64 & & Group-B vs Group-C & 0.129 \\
\hline \multirow[t]{3}{*}{ Mental Composite } & Group-A & 24 & 70.54 & $<0.0001$ & Group-A vs Group-B & 0.149 \\
\hline & Group-B & 54 & 57.31 & & Group-A vs Group-C & $<0.0001$ \\
\hline & Group-C & 22 & 11.91 & & Group-B vs Group-C & $<0.0001$ \\
\hline
\end{tabular}

*P value is calculated by Kruskal-Wallis $\mathrm{H}$ test

${ }^{*} P$ value is calculated by Bonferroni post-hoc test

Table 5 Comparison between patient general data of group C before and after improvement of kt/v

\begin{tabular}{|c|c|c|c|c|c|}
\hline & & $\mathrm{N}$ & Mean \pm SD & Median (IQR) & ${ }^{*} p$ value \\
\hline \multirow[t]{2}{*}{ Pre-session weight (kg) } & Before & 22 & $79.09 \pm 13.99$ & $79.00(66.75-87.25)$ & 0.031 \\
\hline & After & & $78.77 \pm 14.02$ & $79.00(66.50-87.25)$ & \\
\hline \multirow[t]{2}{*}{ Post-session weight (kg) } & Before & 22 & $75.82 \pm 13.91$ & $76.00(64.00-85.00))$ & 0.104 \\
\hline & After & & $75.91 \pm 13.99$ & $76.00(64.00-85.37)$ & \\
\hline \multirow[t]{2}{*}{ Interdialytic weight gain (Kg) } & Before & 22 & $3.21 \pm 0.59$ & $3.00(3.00-4.00)$ & 0.025 \\
\hline & After & & $2.86 \pm 0.58$ & $3.00(2.5-3.00)$ & \\
\hline
\end{tabular}

*P value is calculated by paired t test

Table 6 Paired comparison of dialysis duration and parathyroid hormone in group $\mathrm{C}$ before and after improvement of $\mathrm{kt} / \mathrm{v}$

\begin{tabular}{lcclc}
\hline & N & Median (IQR) & \multirow{2}{*}{ *P value } \\
\cline { 3 - 4 } & & Before & After & \\
\hline $\begin{array}{l}\text { Dialysis session } \\
\text { duration (hrs) }\end{array}$ & 22 & $3.00(3-3)$ & 4.00 & $<0.001$ \\
PTH (pg/dl) \# & 22 & $784.50(343.50-979.50)$ & $\begin{array}{l}632.00(343.50- \\
873.75)\end{array}$ & 0.002 \\
& & & & \\
\hline
\end{tabular}

*P value was computed by Wilcoxon Signed Ranks test

(controlled also to PTH, hemoglobin), it didn't show any significance $(r=-0.168, p=0.068)$ (Table 9).

\section{Discussion}

ESRD is an important problem gradually increasing worldwide [1]. It has a considerable impact on the functional status and quality of life (QOL) perceived by the patient. Even in relatively early stages of chronic kidney disease, it may be mainly due to accumulation of uremic toxins which result in uremic symptoms such as dry skin, sleep difficulty, itching, numbness/tingling, decreased interest in sex, and bone/joint pain which may interrupt daily-life activities of patients [19]. HD is the most commonly used treatment option for this stage [2] a method that removes large amounts of uremic toxins with expected improvement of quality of life. However, hemodialysis, in itself, can lead to significant changes in patients' lifestyle and may possibly impair its quality [3]. This may be due to hemodialysis related complications as muscle cramps, pruritus, anorexia and access problems.

The kidney Disease Outcomes Quality Initiative (K/ DOQI) recommended monitoring the Health Related Quality of Life (HRQOL) for all patients with renal disease [20]. Although there are several standard questionnaires available for assessment of quality of life, the KDQOL-SF is the most commonly used one. It has many advantages compared to other instruments; it has been tested in several populations with kidney disease, has both general and specific modules to assess chronic kidney disease, can be used both for patients on HD and peritoneal dialysis, has questions about the sexual area, and can be self-applied or applied by an interviewer [13]. In a multitude of research, a strong correlation between HD dose and clinical outcomes has been described, and dialysis adequacy is now considered a strong predictor for morbidity and mortality of ESRD patients treated with regular hemodialysis [21]. Dialysis adequacy and optimal dosing can be assessed utilizing several techniques and 
Table 7 comparison between patient laboratory data of group C before and after improvement of kt/v: $(n=22)$

\begin{tabular}{|c|c|c|c|c|c|}
\hline & & $N$ & Mean \pm SD & Median (IQR) & $p$ value* \\
\hline \multirow{2}{*}{ Pre-session Urea (mg/dl) } & Before & 22 & $129.0 \pm 35.17$ & $128.50(98.75-156.00)$ & 0.668 \\
\hline & After & & $132.00 \pm 20.43$ & $133.00(121.25-147.00)$ & \\
\hline \multirow[t]{2}{*}{ Post-session Urea (mg/dl) } & Before & 22 & $56.36 \pm 17.32$ & $59.50(40.00-68.50)$ & 0.001 \\
\hline & After & & $43.73 \pm 6.73$ & $46.50(39.75-48.25)$ & \\
\hline \multirow[t]{2}{*}{ Serum calcium (mg/dl) } & Before & 22 & $8.93 \pm 0.88$ & $9.00(8.33-9.45)$ & 0.322 \\
\hline & After & & $8.75 \pm 0.79$ & $8.9(8.03-9.43)$ & \\
\hline \multirow[t]{2}{*}{ Serum phosphorus (mg/dl) } & Before & 22 & $5.37 \pm 0.75$ & $5.40(4.88-6.03)$ & $<0001$ \\
\hline & After & 22 & $4.06 \pm 0.59$ & $4.00(3.75-4.50)$ & \\
\hline \multirow[t]{2}{*}{ Serum albumin (g/dl) } & Before & 22 & $3.28 \pm 0.46$ & $3.20(2.98-3.50)$ & 0.329 \\
\hline & After & & $3.23 \pm 0.49$ & $3.15(2.88-3.43)$ & \\
\hline \multirow[t]{2}{*}{ Hemoglobin (g/dl) } & Before & 22 & $9.17 \pm 0.78$ & $9.10(8.50-9.73)$ & $<0001$ \\
\hline & After & & $10.90 \pm 0.88$ & $10.00(9.50-10.80)$ & \\
\hline \multirow[t]{2}{*}{$\mathrm{Kt} / \mathrm{V}$} & Before & 22 & $0.99 \pm 0.12$ & $1.03(0.96-1.08)$ & $<0001$ \\
\hline & After & & $1.31 \pm 0.06$ & $1.32(1.25-1.35)$ & \\
\hline
\end{tabular}

*P value is calculated by paired $t$ test

Table 8 Analysis of the subscales of the questionnaire in group C before and after improvement of kt/V

\begin{tabular}{|c|c|c|c|c|}
\hline \multirow[t]{2}{*}{ Subscales of the questionnaire } & \multirow[t]{2}{*}{$\mathbf{N}$} & \multicolumn{2}{|l|}{ Median(IQR) } & \multirow[t]{2}{*}{$P$ value* } \\
\hline & & Before & After & \\
\hline Symptoms / problems list & 22 & $39.58(35.42-45.33)$ & $71.88(41.67-79.17)$ & $<0.001$ \\
\hline Effect of kidney disease & 22 & $50.00(46.09-53.13)$ & $59.38(50.00-68.75)$ & 0.003 \\
\hline Burden of kidney disease & 22 & $31.25(25.00-31.25)$ & $50.00(31.25-62.50)$ & 0.001 \\
\hline Work status & 22 & $50.00(50.00-50.00)$ & $50.00(0.00-50.00)$ & 0.248 \\
\hline Cognitive function & 22 & $73.33(66.67-73.33)$ & $80.00(71.67-86.67)$ & 0.003 \\
\hline Quality of social interaction & 22 & $46.67(38.33-53.33)$ & $66.67(40.00-73.33)$ & 0.002 \\
\hline Sleep & 22 & $35.00(27.50-40.63)$ & $62.50(38.13-70.00)$ & 0.001 \\
\hline Overall health & 22 & $30.00(20-30)$ & $70.00(30.00-70.00)$ & 0.001 \\
\hline Patient satisfaction & 22 & $50.00(45.83-54.17)$ & $41.66(33.33-50.00)$ & 0.115 \\
\hline Physical function & 22 & $32.50(25.00-35.00)$ & $45.00(33.75-55.00)$ & 0.002 \\
\hline Role limitation due to physical function & 22 & $10.00(0.00-50.00)$ & $25.00(25.00-50.00)$ & 0.415 \\
\hline General health & 22 & $55.00(45.00-11.25)$ & $47.50(40.00-55.00)$ & 0.060 \\
\hline Pain & 22 & $12.50(10.00-22.50)$ & $45.00(20.00-55.63)$ & 0.001 \\
\hline Emotion well-Being & 22 & $38.00(32.00-44.00)$ & $60.00(40.00-68.00)$ & 0.001 \\
\hline Role limitations due to emotional problems & 22 & $33.33(0.00-33.33)$ & $33.33(25.00-66.67)$ & 0.194 \\
\hline Social function & 22 & $25.00(12.50-37.50)$ & $50.00(25.00-62.5 .00)$ & 0.002 \\
\hline Energy & 22 & $25.00(20.00-26.50)$ & $45.00(23.75-55.00)$ & 0.001 \\
\hline Physical composite & 22 & $32.49(29.30-36.90)$ & 36.55 (33.99-41.22) & 0.042 \\
\hline Mental composite & 22 & $28.05(24.66-32.21)$ & $35.40(27.49-46.86)$ & 0.002 \\
\hline
\end{tabular}

${ }^{*} P$ value was computed by by Wilcoxon Signed Ranks test

Table 9 Partial correlation of $\mathrm{Kt} / \mathrm{v}$ with some subscales of KDQOL-SF ${ }^{\mathrm{TM}}$ questionnaire (Control variables are PTH, hemoglobin)

\begin{tabular}{llll}
\hline & Work status & $\begin{array}{l}\text { Physical } \\
\text { composite }\end{array}$ & Mental composite \\
\hline $\mathrm{Kt} / \mathrm{vr}$ & -0.168 & 0.304 & 0.587 \\
$\mathrm{p}$ & 0.068 & 0.002 & $<0.0001$ \\
\hline
\end{tabular}

methods; among which, the Kt/v determined by single pool urea kinetic modeling, is the most frequently used and preferred method for the numerical expression of dialysis dose and or adequacy, as it is more specific and accurate [22]. A minimum Kt/V units per dialysis session of 1.2, carried out 3 times per week, has been recommended as an acceptable target; a recommendation that 
was essentially advocated by the well-conducted HEMO study [23].

In Egypt, especially in our locality, many physicians looking after hemodialysis patients are interested mainly in the clinical and laboratory data of their patients, paying little attention to the patients' emotional satisfaction and their quality of life. The present study was planned with a main focus of assessment of HRQOL among ESRD patients treated with hemodialysis in relation to different variables, especially the adequacy of dialysis relying on the calculated kt/V.

So, this work was carried out on 100 ESRD patients ( $51 \%$ males; with mean age of $48 \pm 5.89$ ), maintained on regular hemodialysis three times weekly. Dialysis adequacy was assessed by single pool Kt/V, and KDQOL-SF questionnaire was utilized for determining the quality of life. Taking KDIGO (2012) guidelines for anemia in ESRD in consideration, nearly half of patients in the current study fulfilled the target hemoglobin [24], while $45 \%$ of the patients were within the recommended standard levels of serum calcium according to KDIGO (2009) guidelines and one fifth suffered from low serum calcium [25]. Regarding serum phosphorus, utilizing the recommended values of KDIGO guidelines, nearly a quarter of the patients had hyperphosphatemia, while hypophosphatemia was encountered less frequent. On the other hand, more than one third of the patients had hyperparathyroidism by the previous reference criteria. Hypoalbuminemia afflicted many patients in the present study; this might be due to anorexia, malnutrition and inflammation which are common findings in HD patients [26, 27].

The baseline Kt/v of the majority of patients in the current study, with a mean value of 1.4, was within the target recommendation by the National kidney foundation Disease Outcomes Quality Initiative [20]clinical practice guidelines, $>1.2$, which reflects a reasonable patient care service. However, recent guidelines recommended higher value $>1.4$ which reflect more interest of the international community in improving the dose of dialysis that could have positive impacts on patients quality of life [28].

The physical domain includes three subscales; namely, physical function, role limitation due to physical function, and bodily pain. In previous studies this domain was reported to be a significantly associated predictor of both death and hospitalization [29,30]. This argues for the importance of improving the physical component in hemodialysis patients; a change that is expected to effectively decrease the risk of death and recurrent hospitalization for these patients with less cost on the community [30].

In the current study each of the three subscales of the physical domain had a score of below an average of 50 , suggesting that these patients are suffering from poor physical performance. This is in agreement with the corresponding findings reported for the European and American patients in DOPPS study [31]. However, in the latter study the Japanese patients had a score which was above the previously mentioned average, which are thought to reflect their better awareness of the clinical problems and possibly denoting better health education conducted by their caring staff.

Many studies have proposed several points for improving the component of the physical domain. Physical exercise has been the focus of interest in a good body of literature [32-34]. In a systematic review of 29 clinical trials, improvement of the physical condition following the use of aerobic training was observed [35]. The importance of physical exercise not only has a valuable effect on the physical component of QOL, but also improves cardiopulmonary fitness, anemia, hyperlipidemia, chronic inflammation, blood pressure, insulin resistance, anxiety, depression and adequacy of dialysis [36, 37]. Although the physical exercise in hemodialysis patients has many beneficial effects and it should be mandatory not optional for patients with ESRD, exercise programs are still not a part of routine clinical practice in many countries [38].

On the other hand, the mental domain includes social function, role limitation due to mental function and general mental health. The first two subscales, in the present study, scored above 50 while the third one (mental health) approached 100 which represents the maximum score for this subscale. These findings are in accordance with the corresponding findings of Czyzewski.et.al., 2014 and the DOPPS study $[17,30]$. The mental status of the hemodialysis patients is intimately linked to the social status which is expected to be badly changed by their illness and inability to work and get suitable financial coverage. These patients might consequently lose their self-esteem and feel handicapped. Moreover, hemodialysis itself may cause mood and sleep disorders which would result in more mental suffering, imposing much burden on the caring nephrologists and dialysis staff.

Important points to improve mental status should include training of patients to talk with their doctors whenever they feel down, anxious, worried, nervous or fearful. This may help to assess their problems and start to plan for solutions. Group therapy is another successful way that can improve mental health as not only has a self-confidence effect, but also it enables the patients to feel that they are not alone in their struggle with dialysis [39]. Other ways of intervention may include using antidepressant drugs which should be prescribed in the proper modified doses by caring physicians [40]. More ideas in this respect may comprise utilizing entertainment tools in dialysis wards, such as televisions, radio 
and magazines, recreational group outings, and frequently improving the decoration of dialysis rooms.

In addition to the above-mentioned subscales within the mental and physical domains, two other subscales, namely, energy and general health perception are expected to be reflected on both of them. In the current study, both energy and general health perception showed an average or even below average scores; findings that are in parallel with those reported in a study by Merkus and colleagues, 2017 [41].

$\mathrm{Kt} / \mathrm{v}$, determined by single pool urea kinetic modeling, is the most frequently used and preferred method for the numerical expression of dialysis dose and or adequacy, as it is more specific and accurate [42]. In the current study Kt/V was utilized to divide the patients into three groups of highly adequate, intermediately adequate and inadequate, for the purpose of analytical comparisons, relying on a $\mathrm{Kt} / \mathrm{V}$ cut points of 1.2 and 1.5. A similar way of subdividing the patients relying on cut-off values of $\mathrm{Kt} / \mathrm{V}$ was also adopted by many previous groups of researches. Akhil Babu et al. [43], divided his studied patients into two groups of adequate and inadequate dialysis efficiency, utilizing a $\mathrm{Kt} / \mathrm{V}$ value of 1.4 as a separator. While two other studies [44, 45] divided their patients according to a $\mathrm{Kt} / \mathrm{V}$ of $<1.2$ and $\geq 1.2$.

In the present work, the group with highest $\mathrm{Kt} / \mathrm{V}$ was noticed to have a mean dry weight which is higher than that of the other two groups; an explanation of that may be related to their better dialysis adequacy leading to lower GIT problems, better appetite, lower inflammation and higher anabolism [46]. However, following improvement of the dialysis adequacy for 3 months, the mean dry weight did not show significant changes. Lack of putting on weight in this situation could probably reflect insufficient period of observation; had they been given more time with the better $\mathrm{Kt} / \mathrm{V}$ could have been translated into better body building and more weight gain.

Adequacy of dialysis is related to many factors; for example, duration and frequency of dialysis sessions, dialyzer size and its characteristics, dialysate and blood flow rates, and nature of vascular accesses and whether or not blood recirculation existed [47]. In the current study, the group of poorest dialysis adequacy was noticed to ask for termination of their sessions ahead of time. This noncompliance in sticking to the proper session duration was usually also associated with low compliance regarding diet, salt and water intake, which could be an explanation for their higher interdialytic weight gain in this particular group in comparison to the other two groups. Consequently, increasing the duration of sessions was utilized to improve the dialysis adequacy in this group. Alongside with the significant increase in session duration, there was significant consequent decrease in the interdialytic weight gain.

Having appreciated the positive impact of improving dialysis adequacy on many clinical and biochemical data of patients [6, 48], one would expect welcome effects on the quality of life. In the present research, the quality of life variables were compared among the three groups. These variables were also compared before and after improving dialysis adequacy in group C. All the subscales of the physical domain, except role limitations due to physical function impairment, were found to be significantly low in group C. These low scores were parallel to results reported in similar studies $[49,50]$. Work status was significantly higher in group C ( $p=0.003$ but post hoc analysis didn't show significance between groups). When Work status was correlated to $\mathrm{Kt} / \mathrm{v}$ (controlled to PTH, hemoglobin), it didn't show any significance $(r=-0.168, p=0.068)$. We were not astonished by this non- significant correlation as this subscale was already higher (better) in group $\mathrm{C}$ (lowest Kt/v) than the other 2 groups and improving $\mathrm{Kt} / \mathrm{v}$ wouldn't render a significant change in this group.

The physical domain subscales, apart from the subscales concerning the general health, role limitations due to physical function impairment and work status, were improved after improving of $\mathrm{Kt} / \mathrm{V}$. This improvement in the majority of subscales of the physical domain was associated with improvement in the physical composite score by $12.5 \%$; as this latter score is calculated from all of the above mentioned subscales.

Likewise, all the subscales of the mental and kidney domains were significantly lower in the same group of poorest adequacy; findings confirming previously published data $[49,50]$, some of these subscales did not even show improvement after ameliorating the dialysis adequacy. It is worthwhile to note that the improvement in the mental health composite was two-fold higher than that in the physical health composite. This preferential improvement may possibly be ascribed to repeated patient-doctor interactions during the study which could raise the interest in the concept of quality of life among the patients. This might promote the doctors to pay more attention towards their patients' psychological aspect during reassessment of the KDQol-SF.

One possible explanation for lack of improvement of some subscales after enhancement of the dialysis dose, in spite of the fact that they were better in group A $(\mathrm{Kt} / \mathrm{v}>1.5)$, could be their possible need for more time to achieve their fully expressed response.

Many previous studies examined the association between adequacy of dialysis mostly using $\mathrm{Kt} / \mathrm{V}$ and the HRQOL; some showed no significant association but others showed dissimilar results $[4,41,51]$. These results 
are in contrast with the result in the current study; differences might be due to the small size sample [51], collection of data just after 3 months from the start of dialysis - a time when residual renal function may still be present and a duration that may be too short to have an effect on the quality of life [41]. Another difference could be using urea reduction rate as a single assessment tool for adequacy of dialysis [4].

The present study has the merit of comparing three groups of patients based on $\mathrm{kt} / \mathrm{v}$, one of them above the target and another below the lower target of 1.2, which allowed scrutiny the effects of $\mathrm{kt} / \mathrm{v}$ on different subscales of Qol. Another good point is the paired comparisons of the Qol variables before and after enhancing dialysis adequacy which could envision the benefits of improving the $\mathrm{kt} / \mathrm{v}$ on the patients' perception of their Qol.

Having said that, the present research has got some limitations; firstly, the duration of follow up after improvement of $\mathrm{kt} / \mathrm{v}$ was limited to 3 month - a duration that might not permit full expression of improvement of the quality of life especially in the physical aspect. Secondly, the present study has relied on single measurements of $\mathrm{kt} / \mathrm{v}$ which might not be as accurate indicator of dialysis adequacy as multiple measurements of the same tool over the whole period of observation.

\section{Conclusion}

One would suggest that hemodialysis service providers should have a continuous quality of life assessment plan and make corrective actions when necessary to improve it. Detection and improvement of suboptimal dialysis adequacy is one important point in this aspect as this would have a welcome effect on patients' capabilities as productive members of community.

\section{Acknowledgments}

The authors acknowledge the great efforts and contributions of all staff members of MNDU and all participants in this study since without them, this study would have never been accomplished.

\section{Disclosure}

This work was not funded.

\section{Authors' contributions}

Lamia M, Ahmed M and Nagy AH formulated idea of research. Lamia, Nagy created tables. Dina, Ghada wrote the initial manuscript. Ahmed and Nagy reviewed the manuscript. The author(s) read and approved the final manuscript

\section{Availability of data and materials}

All data analyzed during this study are included in this manuscript.

\section{Declarations}

\section{Ethical approval}

The study was approved by the institutional research board (IRB) of Faculty of Medicine, Mansoura University in accordance with the Declaration of Helsinki. All participants signed a written informed consent before the start of the study.
Consent for publication

Not applicable.

\section{Competing interests}

All the authors declare no conflict of interest in this work.

\section{Author details}

${ }^{1}$ Mansoura Nephrology and Dialysis Unit (MNDU), Mansoura University Hospital, Mansoura, Egypt. ${ }^{2}$ Rheumatology and Immunology Unit, Internal Medicine Department, Mansoura University Hospital, Mansoura, Egypt.

Received: 2 March 2021 Accepted: 23 September 2021

Published online: 07 October 2021

\section{References}

1. Hill NR, Fatoba ST, Oke JL, et al. Global prevalence of chronic kidney disease-a systematic review and meta-analysis. PLoS One. 2016;11(7):e0158765.

2. Makusidi M, Liman H, Yakubu A, Isah M, Abdullahi S, Chijioke A. Hemodialysis performance and outcomes among end stage renal disease patients from Sokoto, North-Western Nigeria. Indian J Nephrol. 2014;24(2):82.

3. Sultania P, Sharma SK, Sharma S. Adequacy of hemodialysis in Nepalese patients undergoing maintenance hemodialysis. J Nepal Med Assoc. 2009;48(173).

4. Ayoub A, Hijjazi K. Quality of life in dialysis patients from the United Arab Emirates. J Fam Community Med. 2013;20(2):106.

5. Held PJ, Port FK, Wolfe RA, et al. The dose of hemodialysis and patient mortality. Kidney Int. 1996;50(2):550-6.

6. Barril G, Besada E, Caro P, et al. News and traditional indications from short daily dialysis: different schemes to optimized ESF response. Hemodial Int. 2004;8(1):85.

7. Johansen $\mathrm{KL}$, Chertow GM, Ng AV, et al. Physical activity levels in patients on hemodialysis and healthy sedentary controls. Kidney Int. 2000;57(6):2564-70.

8. Kimmel PL, Patel SS. Quality of life in patients with chronic kidney disease: focus on end-stage renal disease treated with hemodialysis. Semin Nephrol. 2006:68-79 Elsevier.

9. Bethesda MD. National Institutes of Health, National Institute of Diabetes and Digestive and Kidney Diseases; 2017.

10. Davison SN, Jhangri GS. The impact of chronic pain on depression, sleep, and the desire to withdraw from dialysis in hemodialysis patients. J Pain Symptom Manag. 2005;30(5):465-73.

11. Mujais SK, Story K, Brouillette J, et al. Health-related quality of life in CKD patients: correlates and evolution over time. Clin J Am Soc Nephrol. 2009:4(8):1293-301.

12. Hays RD, Kallich JD, Mapes DL, Coons SJ, Carter WB. Development of the kidney disease quality of life (KDQOLTM) instrument. Qual Life Res. 1994;3(5):329-38

13. Hays RD, Kallich JD, Mapes DL, et al. Kidney disease quality of life short form (KDQOL-SF), version 1.3: a manual for use and scoring, vol. 39. Santa Monica: Rand; 1997.

14. Daugirdas JT, Depner TA, Gotch FA, et al. Comparison of methods to predict equilibrated Kt/V in the HEMO pilot study. Kidney Int. 1997;52(5):1395-405.

15. Korevaar JC, Merkus MP, Jansen MAM, Dekker FW, Boeschoten EW, Krediet RT. Qual Life Res. 2002;1 1(5):437-47.

16. Ware JE, Keller SD, Kosinski M. SF-36 physical and mental health summary scales: Health Assessment Lab Boston; 1994.

17. Czyżewski L, Sańko-Resmer J, Wyzgał J, Kurowski A. Assessment of health-related quality of life of patients after kidney transplantation in comparison with hemodialysis and peritoneal dialysis. Ann Transplant. 2014:19:576-85.

18. ElHafeez SA, Sallam SA, Gad ZM, Zoccali C, Torino C, Tripepi G, et al. Cultural adaptation and validation of the "kidney disease and quality of life-short form (KDQOL-SF ${ }^{\mathrm{TM}}$ ) version 1.3" questionnaire in Egypt. BMC Nephrol. 2012;13(1):170

19. Danquah FVN, Zimmerman L, Diamond PM, Meininger J, Bergstrom N. Frequency, severity, and distress of dialysis-related symptoms reported by patients on hemodialysis. Nephrol Nurs J. 2010;37(6):627-39. 
20. KDOQI. Clinical practice guidelines and clinical practice recommendations for 2006 updates: hemodialysis adequacy, peritoneal dialysis adequacy and vascular access. Am J Kidney Dis. 2006;48(1):1-322.

21. Port FK, Ashby VB, Dhingra RK, Roys EC, Wolfe RA. Dialysis dose and body mass index are strongly associated with survival in hemodialysis patients. J Am Soc Nephrol. 2002;13(4):1061-6.

22. Brenner B, Taal M, Chertow G, Marsden P, Skorecki K, Yu A. Brenner and Rector's the kidney E-book; 2011. p. 2314-8.

23. Eknoyan G, Beck GJ, Cheung AK, Daugirdas JT, Greene T, Kusek JW, et al. Effect of dialysis dose and membrane flux in maintenance hemodialysis. N Engl J Med. 2002;347(25):2010-9.

24. KDIGO. Clinical practice guideline for anemia in chronic kidney disease. Kidney Int. 2012;2:279.

25. KDIGO. Clinical practice guideline for the diagnosis, evaluation, prevention and treatment of chronic kidney disease-mineral and bone disorder (CKD-MBD). Kidney Int. 2009;76(113):1-130.

26. Danielski M, Ikizler TA, McMonagle E, Kane JC, Pupim L, Morrow J, et al. Linkage of hypoalbuminemia, inflammation, and oxidative stress in patients receiving maintenance hemodialysis therapy. Am J Kidney Dis. 2003;42(2):286-94.

27. Sridhar NR, Josyula S. Hypoalbuminemia in hemodialyzed end stage renal disease patients: risk factors and relationships-a 2 year single center study. BMC Nephrol. 2013;14(1):242.

28. Daugirdas JT, Depner TA, Inrig J, Mehrotra R, Rocco MV, Suri RS, et al. KDOQI clinical practice guideline for hemodialysis adequacy: 2015 update. Am J Kidney Dis. 2015;66(5):884-930.

29. DeOreo PB. Hemodialysis patient-assessed functional health status predicts continued survival, hospitalization, and dialysis-attendance compliance. Am J Kidney Dis. 1997;30(2):204-12.

30. Mapes DL, Bragg-Gresham JL, Bommer J, Fukuhara S, McKevitt P, Wikström B, et al. Health-related quality of life in the dialysis outcomes and practice patterns study (DOPPS). Am J Kidney Dis. 2004;44:54-60.

31. Pisoni RL, Gillespie BW, Dickinson DM, Chen K, Kutner MH, Wolfe RA. The Dialysis outcomes and practice patterns study (DOPPS): design, data elements, and methodology. Am J Kidney Dis. 2004;44:7-15.

32. Johansen KL. Exercise in the end-stage renal disease population. J Am Soc Nephrol. 2007;18(6):1845-54.

33. Kosmadakis GC, Bevington A, Smith A, Clapp E, Viana J, Bishop N, et al Physical exercise in patients with severe kidney disease. Nephron Clin Pract. 2010;115(1):C7-c16.

34. Liu Y-M, Yeh M-L, Chung Y-C. Improving physiological and psychological status in a hemodialysis patient: a nursing experience using an exercise training program. Hu Li Za Zhi. 2013;60(5):104.

35. Cheema BSB, Fiatarone Singh MA. Exercise training in patients receiving maintenance hemodialysis: a systematic review of clinical trials. Am J Nephrol. 2005;25(4):352-64.

36. Miller BW, Cress CL, Johnson ME, Nichols DH, Schnitzler MA. Exercise during hemodialysis decreases the use of antihypertensive medications. Am J Kidney Dis. 2002;39(4):828-33.
37. Painter P. Physical functioning in end-stage renal disease patients: update 2005. Hemodial Int. 2005;9(3):218-35.

38. Jung T-D, Park S-H. Intradialytic exercise programs for hemodialysis patients. Chonnam Med J. 2011;47(2):61.

39. Davita 2018. website 4 Ways to Help Manage Mental Health and Kidney Disease. https://www.davita.com/education/ckd-life/support/mentalhealth-services-for-people-with-kidney-disease.

40. Hedayati SS, Yalamanchili V, Finkelstein FO. A practical approach to the treatment of depression in patients with chronic kidney disease and endstage renal disease. Kidney Int. 2012;81(3):247-55.

41. Merkus MP, Jager KJ, Dekker FW, et al. Quality of life in patients on chronic dialysis: self-assessment 3 months after the start of treatment. Am J Kidney Dis. 1997;29(4):584-92.

42. Kuhlmann MK, Kotanko P, Levin NW. Haemodialysis: Dialysis prescription and adequacy. In: Feehally J et al's comprehensive clinical nephrology sixth edition, vol. 1082. Edinburgh London new York; 2019. p. 1089.

43. Babu A, Singh J, Ganesh N. A study on adequacy of hemodialysis and quality of life in Esrd patients OSR. J Pharm Biol Sci (IOSR-JPBS). 2018;18:24-30

44. Chen JB, Lam KK, Su YJ, et al. Relationship between Kt/N urea-based dialysis $9 / 1$ adequacy and nutritional status and their effect on the components of the quality of life in incident peritoneal dialysis patients. BMC Nephrol. 2012;13(1):39.

45. El-Sheikh M, El-Ghazaly G. Assessment of hemodialysis adequacy in patients with chronic kidney disease in the hemodialysis unit at Tanta University Hospital in Egypt. Indian J Nephrol. 2016;26(6):398.

46. Stolic R, Trajkovic G, Jovanovic A, et al. Carotid ultrasonographic parameters as markers of atherogenesis and mortality rate in patients on hemodialysis. Vojnosanit Pregl. 2010;67(11):916-22.

47. Locatelli F, Buoncristiani U, Canaud B, Kohler H, Petitclerc T, Zucchelli P. Haemodialysis with on-line monitoring equipment: tools or toys? Nephrol Dial Transplant. 2005;20(1):22-33.

48. Cigarran S, Coronel F, Torrente J, Sevilla M, Baylón J. Risk of inadequate Dialysis dose in hemodialysis patients with high Watson volume. A warning. Hemodial Int. 2004a;8(1):84.

49. Manns BJ, Johnson JA, Taub K, Mortis G, Ghali WA, Donaldson C. Dialysis adequacy and health related quality of life in hemodialysis patients. ASAIO J. 2002:48(5):565-9.

50. Kalender N, Tosun N. Determination of the relationship between adequacy of dialysis and quality of life and self-care agency. J Clin Nurs. 2014;23(5-6):820-8.

51. Morton AR, Meers C, Singer MA, et al. Quantity of dialysis: quality of life-what is the relationship? ASAIO J (American Society for Artificial Internal Organs: 1992). 1996;42(5):M713-7.

\section{Publisher's Note}

Springer Nature remains neutral with regard to jurisdictional claims in published maps and institutional affiliations.
Ready to submit your research? Choose BMC and benefit from:

- fast, convenient online submission

- thorough peer review by experienced researchers in your field

- rapid publication on acceptance

- support for research data, including large and complex data types

- gold Open Access which fosters wider collaboration and increased citations

- maximum visibility for your research: over 100M website views per year

At BMC, research is always in progress.

Learn more biomedcentral.com/submissions 